\title{
Prevalence and molecular identification ofHepatozoon infection inworking dogs of the Sri Lanka Air Force, free-roaming, and privately-owned dogs: an island-wide survey
}

\author{
PS Jayatilaka ${ }^{1}$, RAS Ranatunga ${ }^{2}$, RS Rajakaruna ${ }^{3}$, ADS Fernando ${ }^{4}$ \\ NGRK Naullage ${ }^{1}$, MCK Mallawa, ${ }^{5}$ A Dangolla ${ }^{5}$
}

Introduction and Objectives:Canine hepatozoonosis is a tick-borne protozoan disease caused by Hepatozoon spp. Two species are currently known to infect dogs: Hepatozoon canis and $H$. americanum. Although $H$. canis generally causes a chronic infection with relatively mild clinical alterations compared to $H$. americanum, infections by $H$. canis can be life-threatening.

Methods: An island-wide survey of canine hepatozoonosis infection was carried out, collecting blood samples from dogs in the Sri Lanka Air Force (SLAF) establishments, freeroaming and privately-owned dogs. Giemsa-stained thin blood smears were observed under light microscopy. Microscopically positive samples were subjected to DNA isolation using 18S rDNA gene primers and sequencing. Sampling was carried out from 2016 to 2019. A total of 668 dogs were sampled, comprising 173 dogs in the three SLAF establishments, 115 free-roaming and 90 owned dogs living in proximity to SLAF establishments and 90 freeroaming and 200 owned dogs that were taken to veterinary clinics island-wide.

Results: Only one privately-owned dog (prevalence $=0.15 \%$ ) from the Colombo Pet Clinic was infected with Hepatozoon. It was a two-year-old male Dachshund with clinical signs. Later in 2020, 65 dogs at the Katunayake SLAF establishment were resampled and one dog (prevalence $=1.5 \%$ ) was found infected - a nine-month-old male Labrador with clinical signs. Phylogenetic analysis of the sequence from the privately-owned dog confirmed the Hepatozoon canis and showed that it had close affinities to Indian and Italian species.

Conclusions: Results show a very low prevalence of Hepatozoon infection among dogs in Sri Lanka. However, it is important to examine blood samples of these dogs for parasite DNA as infections may go undetected if only microscopy is used in diagnosis. Especially freeroaming dogs without clinical signs could have microscopically negative low parasitemia and may act as reservoirs of infection.

Keywords: Canine hepatozoonosis, Military Working dogs, Hepatozoon canis

Funding: National Science Foundation (NSF) of Sri Lanka (Grant No. RG/2019/BT/01).

\footnotetext{
${ }^{1}$ Sri Lanka Air Force

${ }^{2}$ Department of Molecular Biology Faculty of Science, University of Peradeniya, Sri Lanka

${ }^{3}$ Department of Zoology Faculty of Science, University of Peradeniya, Sri Lanka

${ }^{4}$ Government Veterinary Surgeon, Wennappuwa, Sri Lanka

${ }^{5}$ Department of Veterinary Clinical Studies, Faculty of Veterinary Medicine and Animal Science, University of Peradeniya, Sri Lanka
}

Address for correspondence: Dr PS Jayatilaka Telephone: +94717777865 Email:vet_sam@yahoo.com

(iD https://orcid.org/0000-0001-8985-9687 